\title{
Can communication media improve the KAP status of frontline health workers? A cross-sectional study on $\mathrm{MCH}$ indicators from Assam, India
}

\author{
Lipi B Mahanta ${ }^{1}$, Manisha Choudhury ${ }^{2}$, Sikha Borthakur ${ }^{3}$, Ritul Bhagabati ${ }^{4}$, Partha Gogoi ${ }^{5}$
}

\begin{abstract}
${ }^{1}$ Associate Professor-I, Center for Computational and Numerical Studies, Institute of Advanced Study in Science and Technology, Assam, India. ${ }^{2}$ State Reproductive and Adolescent Health Consultant, NHM, Assam (UNICEF support); ${ }^{3}$ Behaviour Change Communication (BCC) Expert; Office of the Mission Director, National Health Mission (NHM), Assam, India. ${ }^{4}$ Senior Copy Editor, News 18, Axom, North East, Guwahati, Assam, India. ${ }^{5}$ Regional Director, North East, Office of the Regional Director, Health and Family Welfare, Government of India, Assam, India.
\end{abstract}

\section{Abstract}

Frontline health workers are now key component in any public health delivery system, providing essential information on basic components of health, nutrition and sanitation for improving the utilization of primary health care delivery system by the community. This study was conducted to find out the Knowledge Attitude and Practices (KAP) of Accredited Social Health Activist (ASHA) workers on maternal health and child health components, service delivery system and to find out the association of different communication media on KAP status regarding above components. It is a cross-sectional study. Data was collected using a pre-tested structured questionnaire from 264 ASHA workers from 8 blocks of 5 districts of Assam selected randomly. Multiple regression analysis has been used to associate various findings and variables. The overall KAP status of most of the ASHAs (72\%) was found to be 'Good'. It was observed that $70.45 \%$ had good KAP regarding maternal health components, $82.2 \%$ had good KAP on child health components, and $78.79 \%$ on common diseases. Communication media like TV, radio and mobile have also been found to have significant effect on certain KAP by ASHAs. Most of the ASHAs have been found competent in some aspects of health and nutrition components which have direct impact on child survival and maternal health. But at the same time average to poor knowledge level have been seen in some other important indicators. Hence proper training and timely re-orientation and information sharing through different suitable medium would be helpful for improving the KAP status of ASHAs.

Keywords: KAP, Frontline wealth workers, Communication media, MCH, India.

\section{Introduction}

Frontline health workers are the first and often the only point of contact to the health care system for millions of people in the rural populations as they deliver a range of lifesaving interventions to prevent illness, death and disability among vulnerable groups. The developing world has experienced notable reduction in maternal, child and infant mortality in recent decades. Millions of people in impoverished countries are alive today because a frontline health worker was by their side during delivery of newborn followed by successive vaccinations and for adopting healthy behaviours like breastfeeding, hand washing, birth spacing, and sleeping under a mosquito net. ${ }^{1}$

The Accredited Social Health Activist (ASHA), the flagship programme under the National Rural Health Mission (NRHM), India, is the latest of its kind that is operational throughout the country. ${ }^{2}$ Having a community health worker for every village with a population of 1000 was one of the key strategies under the NRHM. These ASHA workers should preferably be female, in the 25-45 years age group, and have a qualification of at least eighth class. ${ }^{3}$ ASHA represents the pivotal part in the whole design and strategy of the NRHM, which, in turn, is a

\section{Practice Points}

- The overall KAP status of most of the ASHAs was found to be 'Good'.

- Communication media like $\mathrm{TV}$, radio and mobile have significant effect on certain KAP by ASHAs.

- Most of the ASHAs were found competent in some aspects of health and nutrition components which have direct impact on child survival and maternal health.

- Proper training and timely re-orientation and information sharing through different suitable medium would be helpful for improving the KAP status of ASHAs.

- Capacity building of ASHAs or re-orientation on different schemes and programmes will be helpful for updating KAP status of ASHAs.

critical initiative of the central government to fulfil its promise on inclusive growth. ${ }^{4}$

Frontline health workers do not need to be highly

Correspondence: Ms. Manisha Choudhury, State Reproductive and Adolescent Health Consultant (UNICEF Support), Office of the Mission Director, National Health Mission (NHM), Assam, Christianbasti, Guwahati-781005, Assam, India. Email: manishachoudhury07@gmail.com. 
educated to be successful in their job. It has been seen that in many developing countries, health workers with basic education plus several weeks of well-designed training, followed by supportive supervision, can master the skills needed to diagnose and treat common illnesses, promote lifesaving health practices and counsel families about family planning, nutrition and hygiene. ${ }^{1}$ But despite giving training to ASHAs, lacunae still persists in their knowledge regarding various aspects of child morbidity and mortality. ${ }^{4}$ Moreover, as per other studies, ASHA workers were found to have poor knowledge in some aspects of maternal, child health and related areas. ${ }^{5-7}$ Further, the services provided by them were not consistent enough to have substantial impact and the quality of services was poor. ${ }^{8}$ This lacuna can be improved by some other means apart from training. Communication media plays an important role in disseminating any message to the community for better understanding and practice. For improving the Knowledge, Attitude and Practice (KAP) of any frontline workers, behaviour change communication through a proper media is utmost necessary. It can help in improving the knowledge so as to practice through continuous hammering if strategically used.

The performance of ASHAs is, therefore, crucial for the success of NRHM and hence of the inclusive growth strategy of the government in India and to achieve the health related millennium development goals such as infant mortality rate (IMR), maternal mortality rate (MMR); as well as control of specific diseases, and improvement of nutrition status of children and mothers. ${ }^{9}$ Activity of ASHA is one of the key components in the NRHM. They provide information to the community on determinants of health such as nutrition, basic sanitation, hygienic practices, healthy living and working condition, information on existing health services and need for timely utilization of health and family welfare services. They can play an important role in identifying child morbidity at the earliest and help in improving their health status. Therefore it is important to assess the level of knowledge regarding health and health aspects in this workers. ${ }^{3}$ For improving their level of knowledge, ASHAs have been provided with different module training along with IEC (Information Education \& Communication) materials. As per the latest data there are more than 8.9 Lakh ASHAs in the country covering 18 states where NRHM is launched. ${ }^{10}$

In Assam the figure is around 30,000. Under NRHM, Assam a radio programme was launched for the ASHAs in 2007 and they have been provided with a radio set so that they can get the important health messages and know the new services/schemes on time. As ASHA is the first contact point for the community for availing primary health care services, the KAP of ASHA has positive impact in changing behaviour towards accepting primary health care services. In this scenario, an attempt has been made to know the KAP status of ASHAs in various indicators and impact of different communication media on this. The main aim and objectives of the study are to find out the KAP of
ASHA workers on maternal and child health components and to find out the association of different communication media on KAP status regarding these two components.

\section{Materials and methods}

The study was conducted during 2009 and 2010. The study subjects comprised of 264 numbers of ASHA workers who were interviewed during monthly review meetings at block level. The sample size was obtained using the standard sample size determination formula, on assigning the margin of error as $6 \%$ and taking $95 \%$ confidence level. There was no specific inclusion criteria for the study, as all ASHA workers currently in service during the study period were considered as population size. This was a cross-sectional study carried out in 5 districts of Assam. Total 8 health blocks were randomly selected from the 5 districts which were again randomly selected based on geographic locations, representing both upper and lower part of Assam, by simple random sampling. Data was collected regarding their KAP about topics, like quality antenatal check-up and care (ANC), dangers signs of pregnancy, breastfeeding, complimentary feeding, immunization, vitamin -A supplementation, diarrhoea, essential new born care, malaria, personal hygiene etc.

\section{Study tools and techniques of data analysis:}

All information was obtained by using a pre-tested structured questionnaire with multiple answer choices. Data regarding the socio-economic, demographic and environmental condition of their house along with available communication medium in their houses were also taken to find out the association of those indicators on KAP of the frontline health workers. Then data were compiled in excel sheet and calculations and data analysis was done with the help of SPSS-14 version. Multiple regression analysis has been used to associate various findings and variables. If the question were of important nature higher weights were assigned to the subsequent response. Thus, the KAP of ASHAs on health related indicators have been categorised as 'Good', 'Average' and 'Poor' according to the specifications as shown below:

- Weightage $1=$ Normal (Category=Good; Specification $=$ correct answers $\geq 60 \%$ )

- Weightage 2 = Important (Category=Average; Specification=correct answers between $40-59 \%$ )

- Weightage 3 = Very Important (Category=Poor; Specification $=$ correct answers $<40 \%$ )

As no experiment was done on the respondents or none of the respondents were deprived from any benefit ensuing from the study, no ethical permission was required. But for conducting the study, permission from the top authority (Mission Director, National Rural Health Mission, Assam) was taken. Moreover, all participants were explained the motive of the study prior to the filling up of the questionnaire and consent of the respondents was taken as a part of the formal study. 


\section{Results}

The socio-economic and environmental profile of the ASHA workers has been shown in Table 1. Most of the ASHAs are in the age group of 30-40 years (43.2\%) followed by $20-29$ years $(35.2 \%)$ though there are quite a large group of ASHAs who fall in the age group above 40 years $(21.6 \%)$. Although the minimum qualification of ASHA worker is class eight, it was found that majority of them $(62.9 \%)$ had above class X level of education. About $29.2 \%$ of ASHA workers were found tobe in between class VIII to X. Regarding type of housing, majority of ASHAs (85.2\%) had kachcha house (earthen house).

The source of drinking water for majority (83.3\%) was found to be hand pump. Regarding presence of toilet, majority $(84.8 \%)$ had their own toilet. But, out of those, only $23.1 \%$ had sanitary toilet. Regarding communication medium, it has been found that, around $94.7 \%, 62.5 \%$ and $25.8 \%$ of ASHA workers had radio, mobile phone and TV respectively.

\section{Knowledge level of ASHAs on behavioural attitude during home visit}

The behavioural attitude of ASHAs was also tried to be captured during the study as this is one of the important components in Behaviour Change Communication (BCC). It was found that $83.7 \%$ of ASHAs knew that they should greet first by saying Namaskar (a standard

Table 1: Socio-economic and Environmental profile of respondents

\begin{tabular}{|c|c|}
\hline Parameters & Respondents (\%) \\
\hline \multicolumn{2}{|l|}{ Age group } \\
\hline $20-29$ & $93(35.2 \%)$ \\
\hline $30-40$ & $114(43.2 \%)$ \\
\hline$>40$ & $57(21.6 \%)$ \\
\hline \multicolumn{2}{|l|}{ Education level } \\
\hline Below class 8 & $21(8.0 \%)$ \\
\hline Class 8 to 10 & $77(29.2 \%)$ \\
\hline Above class 10 & $166(62.9 \%)$ \\
\hline \multicolumn{2}{|l|}{ Type of house } \\
\hline Kachcha & $224(85.2 \%)$ \\
\hline Pucca & $39(14.8 \%)$ \\
\hline \multicolumn{2}{|c|}{ Source of drinking water } \\
\hline Well & $39(14.8 \%)$ \\
\hline Handpump & $220(83.3 \%)$ \\
\hline River & $5(1.9 \%)$ \\
\hline \multicolumn{2}{|l|}{ Presence of latrine } \\
\hline Yes & $224(84.8 \%)$ \\
\hline No & $40(15.2 \%)$ \\
\hline \multicolumn{2}{|c|}{ If yes, type of latrine } \\
\hline Kachcha & $162(61.4 \%)$ \\
\hline Sanitary & $61(23.1 \%)$ \\
\hline \multicolumn{2}{|l|}{ Presence of TV } \\
\hline Yes & $68(25.8 \%)$ \\
\hline No & $196(74.2 \%)$ \\
\hline \multicolumn{2}{|l|}{ Presence of Radio } \\
\hline Yes & $250(94.7 \%)$ \\
\hline No & $14(5.3 \%)$ \\
\hline \multicolumn{2}{|l|}{ Presence of mobile } \\
\hline Yes & $165(62.5 \%)$ \\
\hline No & $99(37.5 \%)$ \\
\hline
\end{tabular}

Indian greeting) during home visit. About $75.4 \%$ of ASHAs knew the way of communication during home visit, though the visits were targeted visits to the beneficiary home only.

\section{Knowledge level of ASHAs on antenatal care, new-born} care and IYCF

Quality Antenatal Check up (ANC) reduces the risk of complications during pregnancy. Each pregnant woman should do at least four ANCs during her pregnancy till child birth. ASHAs are expected to know the basic care during pregnancy and about the quality antenatal checkups. Regarding the Knowledge level on Antenatal care, (Table 2) it was found that $44.7 \%$ of ASHAs knew the time of first ANC. Total 52.3\% think that first ANC is to be done within 5 months of pregnancy, not as soon as pregnancy is confirmed. But almost all ASHAs (98.1\%) knew about the minimum numbers of ANCs to be done during pregnancy period. Regarding quality of ANC, around $96.2 \%$ ASHAs knew that at least 100 IFA tablets are to be taken and around $93.9 \%$ knew about the number of TT injections to be taken during pregnancy. Similarly most of them knew about adequate amount of rest and tests to be done during pregnancy. But regarding danger signs of pregnancy, only $21.2 \%$ ASHAs knew that pain before 8 months of pregnancy could be a danger sign. Similarly, only $11 \%$ of the respondents knew that any type of discharge from vagina could be a danger sign. It was observed that the ASHAs knew about institutional delivery being the best form of delivery with $53.4 \%$ of them reporting that Institutional delivery after completing all required ANCs is the best form of delivery and $43.6 \%$ of them answering that institutional delivery is best form of delivery, though they didn't mention about the ANCs. Therefore it can be concluded that around 97\% ASHAs knew about importance of institutional delivery.

Regarding the New born care and Infant and Young Child Feeding (IYCF) practices, it was observed that ASHAs have good knowledge on the subject (Table 2). About 89.4\% ASHAs had KAP regarding early initiation of breastfeeding just within half an hour to one hour after birth and same percentage knew about the exclusive breastfeeding till 6 months. Moreover, 95.5\% ASHAs knew the importance of Colostrums for the new -born and they tried to motivate the mothers and caregivers to follow the same. But regarding continued breastfeeding only $34.5 \%$ ASHAs knew that it should be done till 2 years and beyond. Almost same percentage i.e. $35.2 \%$ knew that continued breastfeeding is till 6 months. $83.3 \%$ ASHAs had knowledge on timely initiation of complementary feeding after 6 months of age. About $78.4 \%$ of ASHAs knew about Kangaroo care.

Knowledge level of ASHAs on vaccination/ immunization, common illnesses and their treatment

The KAP of ASHAs on Full immunization was found to be good (Table 3). About $92 \%$ of ASHAs knew that full immunization means completing all vaccines till the age of 9 months. But only $47 \%$ of ASHAs could answer the 
number of Vitamin-A doses to be provided to the children till five years of age, i.e. 9 doses in 6 months interval from 9 months of age. $41.3 \%$ knew that it is 5 doses. Therefore, it was observed that, most of the ASHAs didn't give importance to Vitamin A though it is one of the important intervention for reducing child malnutrition. This part should be strengthened with proper IEC -BCC activities well in advance before the biannual Vitamin-A rounds.

Regarding diarrhoea, $95.8 \%$ of ASHAs knew the symptoms of diarrhoea. $81.4 \%$ knew how to prepare ORS. Regarding malaria, $80.3 \%$ knew the causes and symptoms malaria and how to detect it through RDK (Rapid Diagnostic Kit). 95.5\% of respondents knew that blood test is to be done to detect malaria. 54.4\% ASHAs knew all sorts of ways for prevention of malaria from using mosquito net to DDK, though $36.4 \%$ ASHAs knew only one way of prevention of malaria, i.e. use of mosquito net (Table 3).

\section{Overall KAP of ASHAs on different indicators}

Overall KAP of ASHAs on health related indicators have been categorised as 'Good', 'Average' and 'Poor' as categorized above. Further weights were associated with the questions depending upon their importance as follows: $1=$ Normal, $2=$ Important and $3=$ Very important. It was observed that amongst ASHA workers, $70.45 \%$ had good KAP regarding Maternal Health components, $82.2 \%$ had good KAP on Child Health components, and $78.79 \%$ on Common diseases (Table 4).

Association of communication media, education and age on KAP status of ASHA

During the study, association between KAP of ASHAs and educational level, communication media like TV, radio and age of ASHAs have been statistically

Table 2: Knowledge level of ASHAs on antenatal care, newborn care and IYCN

\begin{tabular}{|c|c|c|c|}
\hline Query & Respondents & Query & Respondents \\
\hline 1. When is first ANC to be done? & & No & $208(78.8 \%)$ \\
\hline As soon as pregnancy is confirmed* & $118(44.7 \%)$ & 13. Is bleeding a danger sign during pregnancy? & \\
\hline Within 5 months of pregnancy & $138(52.3 \%)$ & Yes* & $42(15.9 \%)$ \\
\hline After 6 months of pregnancy & $2(0.8 \%)$ & No & $222(84.1 \%)$ \\
\hline After 8 months of pregnancy & $6(2.3 \%)$ & 14. Is itching a danger sign during pregnancy? & \\
\hline 2. Number of times a pregnant women must have ANC & & Yes & $79(29.9 \%)$ \\
\hline 5 times & $5(1.9 \%)$ & No* & $185(70.1 \%)$ \\
\hline 4 times* & $259(98.1 \%)$ & 15. Which is the best form of delivery? & \\
\hline 3. Number of IFA pills a pregnant women must consume & & Hospital/Institutional & $115(43.6 \%)$ \\
\hline 300 & $8(3.0 \%)$ & Home & $8(3.0 \%)$ \\
\hline $100^{*}$ & $254(96.2 \%)$ & Hospital/Institutional after completing all ANCs* & $141(53.4 \%)$ \\
\hline 50 & $2(0.8 \%)$ & 16. Time a new born should be breast fed & \\
\hline 4. Number of TT vaccines during pregnancy & & Within 1 day & $17(6.4 \%)$ \\
\hline 1 & $1(0.4 \%)$ & After 2 days & $5(1.9 \%)$ \\
\hline 2* & $248(93.9 \%)$ & Within half to 1 hour within birth* & $236(89.4 \%)$ \\
\hline 3 & $12(4.5 \%)$ & After 10 hours & $6(2.3 \%)$ \\
\hline 4 & $3(1.1 \%)$ & 17. Is the colostrums best for babies? & \\
\hline 5. Which test is generally not done during ANC & & Yes* & $252(95.5 \%)$ \\
\hline Blood pressure & $46(17.4 \%)$ & No & $12(4.5 \%)$ \\
\hline Stomach examination & $23(8.7 \%)$ & $\begin{array}{l}18 \text {. How many long a baby should be exclusively } \\
\text { breastfed? }\end{array}$ & \\
\hline Anaemia & $38(14.4 \%)$ & 6 months* & $236(89.4 \%)$ \\
\hline Stool examination* & $157(59.5 \%)$ & 1 year & $1(0.4 \%)$ \\
\hline 6. Is rest at day time also required during pregnancy & & 1 month & $8(3.0 \%)$ \\
\hline Yes* & $253(95.8 \%)$ & 2 years & $19(7.2 \%)$ \\
\hline No & $11(4.2 \%)$ & 19. What is kangaroo care? & \\
\hline 7. Is hair loss a danger signal during pregnancy? & & Carry new-born in back sling & $49(18.6 \%)$ \\
\hline Yes & $120(45.5 \%)$ & Family members look after new-born & $8(3.0 \%)$ \\
\hline No* & $144(54.5 \%)$ & $\begin{array}{l}\text { Hold new-born in skin to skin contact with } \\
\text { mother* }\end{array}$ & $207(78.4 \%)$ \\
\hline 8. Is pain before 8 months a danger sign? & & 20. How long the breast feeding to be continued? & \\
\hline Yes* & $56(21.2 \%)$ & At least 2 years* & $91(34.5 \%)$ \\
\hline No & $208(78.8 \%)$ & Up to 6 months & $93(35.2 \%)$ \\
\hline 9. Is any type of discharge from the vagina a danger sign? & & Up to 2 years & $52(19.7 \%)$ \\
\hline Yes* & $29(11.0 \%)$ & Up to 4 years & $28(10.6 \%)$ \\
\hline No & $235(89.0 \%)$ & $\begin{array}{l}\text { 21. When complementary feeding to be started to an } \\
\text { infant? }\end{array}$ & \\
\hline 10. Is excessive sweating a danger sign during pregnancy? & & After 1 year & $34(12.9 \%)$ \\
\hline Yes* & $73(27.7 \%)$ & From 2 months & $10(3.8 \%)$ \\
\hline No & $191(72.3 \%)$ & After 6 months* & $220(83.3 \%)$ \\
\hline 11. Is reduction of red pigment in eye a danger sign? & & $\begin{array}{l}\text { 22. When the first bath to a newborn to be preferably } \\
\text { done? }\end{array}$ & \\
\hline Yes* & $35(13.3 \%)$ & Immediately after birth & $7(2.7 \%)$ \\
\hline No & $229(86.7 \%)$ & 3 days after birth & $58(22.0 \%)$ \\
\hline 12. Is swelling of foot a danger sign during pregnancy? & & 7 days after birth* & $199(75.4 \%)$ \\
\hline 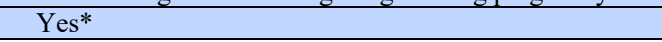 & $56(21.2 \%)$ & & \\
\hline
\end{tabular}


Table 3: Knowledge level of ASHAs on vaccination/ immunization, common illnesses and their treatment

\begin{tabular}{|c|c|}
\hline Query & Responses \\
\hline \multicolumn{2}{|l|}{ What is full immunization? } \\
\hline Immediately after birth & $14(5.3 \%)$ \\
\hline Immunization during delivery & $7(2.7 \%)$ \\
\hline $\begin{array}{l}\text { Completing immunization from birth to } 9 \\
\text { months of the baby (till dose for measles)* }\end{array}$ & $243(92.0 \%)$ \\
\hline \multicolumn{2}{|l|}{$\begin{array}{l}\text { How many Vitamin } A \text { doses to be given to a } \\
\text { child? }\end{array}$} \\
\hline 5 & $109(41.3 \%)$ \\
\hline 7 & $22(8.3 \%)$ \\
\hline $9 *$ & $124(47.0 \%)$ \\
\hline None & $9(3.4 \%)$ \\
\hline \multicolumn{2}{|l|}{ What happens when diarrhoea occurs? } \\
\hline Cold & $2(0.8 \%)$ \\
\hline Cough & $3(1.1 \%)$ \\
\hline Watery latrine* & $253(95.8 \%)$ \\
\hline Fever & $6(2.3 \%)$ \\
\hline \multicolumn{2}{|l|}{ How to prepare ORS? } \\
\hline 1 packet ORS in 1 litre unboiled water & $48(18.2 \%)$ \\
\hline $1 / 2$ packet ORS in 1 litre unboiled water & $1(0.4 \%)$ \\
\hline 1 packet ORS in 1 litre boiled water* & $215(81.4 \%)$ \\
\hline \multicolumn{2}{|l|}{ What is the RDK kit for? } \\
\hline Testing diarrhoea & $9(3.4 \%)$ \\
\hline Testing malaria* & $212(80.3 \%)$ \\
\hline Testing anaemia & $43(16.3 \%)$ \\
\hline \multicolumn{2}{|l|}{ What to do for prevention of malaria? } \\
\hline Use mosquito net & $96(36.4 \%$ \\
\hline Spread DDT in house & $17(6.4 \%)$ \\
\hline Clear water logging & $7(2.7 \%)$ \\
\hline All the above* & $144(54.5 \%)$ \\
\hline \multicolumn{2}{|l|}{ Where to spread DDT? } \\
\hline Inside house boundary* & $124(47.0 \%)$ \\
\hline Outside house boundary & $13(4.9 \%)$ \\
\hline Both the above & $127(48.1 \%)$ \\
\hline \multicolumn{2}{|l|}{ How to detect malaria? } \\
\hline Blood test* & $252(95.5 \%)$ \\
\hline External appearance & $5(1.9 \%)$ \\
\hline With thermometer & $7(2.7 \%)$ \\
\hline
\end{tabular}

examined. Multiple regression was done and the $p$-values showing the association of communication media like TV, radio and age of ASHAs on KAP status of ASHAs has been depicted in Table 5.

\section{Discussion}

Regarding age of the respondents, similar findings were also found, ${ }^{11}$ where majority of ASHAs fall in the age group found in the present study. As per the guidelines of National Rural Health Mission, Ministry of Health \& Family Welfare, Government of India, the age group of ASHAs should be between 25-45 years. ${ }^{10}$ The age group of the present study also falls in the same group though cases above 45 years of ASHAs were also found. Regarding education level, similar findings were also found in Surendranagar ${ }^{5}$ where around $70 \%$ of ASHA workers were found to have secondary level education. Similar finding were also found in Maharashtra. ${ }^{4}$

Regarding knowledge level on antenatal care, new-born care and IYCF, related findings were found, where $86.2 \%$ of ASHA workers had improper knowledge regarding new born care, ${ }^{5} 100 \%$ correct knowledge was observed regarding counselling the exclusive breastfeeding of newborns ${ }^{4}$ and $81.3 \%$ of ASHA workers had knowledge about their responsibilities regarding counselling on antenatal care/ postnatal care, breastfeeding and immunization in Orissa. ${ }^{7}$ Regarding improved knowledge and practice on institutional delivery, this might be due to the Janani Suraksha Yojana (JSY) for which ASHAs are getting their incentives along with the beneficiaries. Even in some other studies, approximately $78.9 \%$ of the beneficiaries of the Janani Suraksha Yojana (JSY) service said that ASHA workers encouraged them to use the service. ${ }^{7}$

Moreover, $100 \%$ of the ASHA workers displayed correct knowledge about the JSY service. ${ }^{4}$ Regarding knowledge on vaccination/immunization, similar findings were reported where $100 \%$ ASHAs know the importance of immunization and actually helping other healthcare staff in carrying out immunization services. ${ }^{4}$ But a contradictory finding was also reported where $68.46 \%$ of frontline workers had lack of knowledge about measles which is one of the important components of full immunization. ${ }^{5}$ Regarding common illnesses and their treatment, related findings were found where nearly $70 \%$ were aware about the causes of diarrhoea but $91.5 \%$ of them had no idea about signs and symptoms of dehydration. In the same contradictory finding, $19.9 \%$ ASHAs did not feel the need for referral of any child with diarrhoea and unable to drink or breast feed. In respect of knowledge on malaria, similar findings were found where approximately $80.77 \%$ knew about signs and symptoms of malaria but $59.23 \%$ among them did not know what to do if the child was suffering from it. ${ }^{5}$

The present study found that age of the respondents have significant effect on the KAP status of few indicators and behaviours like, the first greetings during home visit, importance of IFA consumption during pregnancy, required tests during pregnancy, danger signs of pregnancy, newborn care within first 7 days, number of Vitamin doses and ORS preparation. It was also identified that participants with age group of 25-40 years showed significant positive response on the above -mentioned factors. Other age group didn't show significant effect. The response of age group of 25-45 years of ASHAs was also found to be as per recommendations of National guidelines. ${ }^{10}$ Therefore during selection of ASHAs the age should be considered as per guidelines only.

Similarly, education level was found to have significant positive effect on certain points like the first greetings during home visit, importance of IFA consumption during pregnancy, danger signs of pregnancy,

Table 4: Overall KAP of ASHAs by indicators

\begin{tabular}{|c|c|c|c|c|}
\hline \multirow{2}{*}{$\begin{array}{c}\text { Components } \\
\text { status }\end{array}$} & \multicolumn{4}{|c|}{ Respondents (\%) } \\
\hline & MH & CH & CD & VHND \\
\hline Poor & $\begin{array}{c}30 \\
(11.36 \%)\end{array}$ & $\begin{array}{c}9 \\
(3.41 \%)\end{array}$ & $\begin{array}{c}9 \\
(3.41 \%)\end{array}$ & $\begin{array}{c}47 \\
(17.8 \%)\end{array}$ \\
\hline Average & $\begin{array}{c}48 \\
(18.18 \%)\end{array}$ & $\begin{array}{c}38 \\
(14.39 \%)\end{array}$ & $47(17.8 \%)$ & 0 \\
\hline Good & $\begin{array}{c}186 \\
(70.45 \%)\end{array}$ & $\begin{array}{c}217 \\
(82.2 \%)\end{array}$ & $\begin{array}{c}208 \\
(78.79 \%)\end{array}$ & $\begin{array}{c}217 \\
(82.2 \%)\end{array}$ \\
\hline
\end{tabular}

Keys: MH- Maternal health, CH- Child health, CD- Common diseases, VHND- Village Health and Nutrition Day 
Mahanta et al. - Can communication media improve the KAP status of frontline health workers?

Table 5: Multiple regression table (Beta and $p$ values) association of communication media, education, age on KAP status of ASHA

\begin{tabular}{|c|c|c|c|c|c|}
\hline \multirow{3}{*}{ Dependent Variables } & \multicolumn{5}{|c|}{ Predictors } \\
\hline & \multirow{2}{*}{ Age group } & \multirow{2}{*}{ Education Level } & \multicolumn{3}{|c|}{ Presence of } \\
\hline & & & TV & Radio & Mobile \\
\hline \multirow[t]{2}{*}{ First greetings by ASHA } & .088 & .089 & -.041 & -.066 & -.050 \\
\hline & .056 & .098 & .616 & .667 & .495 \\
\hline \multirow[t]{2}{*}{ Which is to prevented during communication } & .009 & .1 & -.001 & .194 & -.097 \\
\hline & .923 & .373 & .993 & .546 & .532 \\
\hline \multirow[t]{2}{*}{ When is first ANC to be done? } & .033 & -.085 & -.001 & .194 & .097 \\
\hline & .524 & .165 & .993 & .546 & .532 \\
\hline Number of times a pregnant women must have ANC & .002 & -.010 & .033 & -.113 & -.009 \\
\hline & .922 & .707 & .421 & .140 & .804 \\
\hline Number of IFA pills a pregnant women must consume & .032 & .032 & .001 & .051 & -.028 \\
\hline & .050 & .090 & .960 & .345 & .282 \\
\hline Number of TT injections during pregnancy & -.006 & .016 & .060 & .549 & -.060 \\
\hline & .789 & .548 & .145 & .000 & .107 \\
\hline Which test is generally not done during ANC? & -.199 & .059 & -.152 & .019 & .027 \\
\hline & .043 & 606 & .379 & 953 & .862 \\
\hline Is rest at day time also required during pregnancy? & .010 & -.026 & -.006 & .023 & .011 \\
\hline & .561 & .185 & .828 & .679 & .687 \\
\hline Is hair loss a danger sign during pregnancy? & .017 & -.037 & .069 & .160 & .033 \\
\hline & 691 & .453 & .348 & .252 & .620 \\
\hline Is pain before 8 months a danger sign during preg- & -.017 & .040 & .036 & -.067 & -.017 \\
\hline nancy? & .616 & .326 & .552 & .559 & .762 \\
\hline Is any type of discharge from the vagina a danger sign & .022 & -.019 & .069 & -.040 & .010 \\
\hline during pregnancy? & .411 & .537 & .138 & .647 & .809 \\
\hline Is excessive sweating a danger sign during pregnancy? & -.038 & .068 & .024 & .2 & -.115 \\
\hline & .305 & .122 & .714 & .109 & .056 \\
\hline Is reduction of red pigment in eye a danger sign during & .024 & .029 & .013 & .013 & -.031 \\
\hline pregnancy? & .393 & .383 & .801 & .892 & .496 \\
\hline Is swelling of foot a danger sign during pregnancy? & -.060 & .070 & .010 & -.196 & -.128 \\
\hline & .072 & .074 & .860 & .079 & .018 \\
\hline Is bleeding a danger sign during pregnancy? & -.025 & .033 & .028 & -.051 & -.020 \\
\hline & .422 & .356 & .602 & .618 & .687 \\
\hline Is itching a danger sign during pregnancy? & .038 & .041 & .048 & -.094 & -.092 \\
\hline & .324 & .364 & .475 & .465 & .138 \\
\hline Which is the best form of delivery? & .021 & .105 & -.181 & -.104 & .248 \\
\hline & .802 & .275 & .212 & .705 & .061 \\
\hline Is the colostrums best for babies? & -.016 & -.041 & -.017 & .067 & .063 \\
\hline & .399 & .072 & .622 & .3 & .042 \\
\hline Time a new born should be breast fed & -.012 & -.012 & -.154 & -.441 & .211 \\
\hline & .789 & .809 & .046 & .003 & .003 \\
\hline How long a baby should be exclusively breastfed? & .104 & -.057 & .235 & -.103 & -.195 \\
\hline & .134 & .480 & .057 & .656 & .081 \\
\hline What is kangaroo mother care? & .051 & .114 & -.176 & -.128 & .062 \\
\hline & .436 & .137 & .128 & .558 & .556 \\
\hline How long the breast feeding to be continued? & .073 & -.156 & .162 & .443 & -.109 \\
\hline & .373 & .104 & .262 & .106 & .406 \\
\hline When complementary feeding to be started to an & .078 & .073 & -.044 & .334 & -.219 \\
\hline 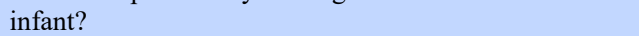 & .167 & .267 & .658 & .077 & .017 \\
\hline When the first bath to a newborn to be preferably done? & .080 & -.066 & -.057 & -.411 & .118 \\
\hline & .052 & .169 & .433 & .003 & .074 \\
\hline Within how many days, a birth certificate can be & -.009 & .096 & -.032 & .303 & .105 \\
\hline acquired free of cost? & .810 & .032 & .637 & .018 & .086 \\
\hline From where a birth certificate can be collected? & -.081 & .045 & .134 & -.140 & .057 \\
\hline & .166 & .514 & .198 & .474 & .548 \\
\hline What is full immunization? & -.053 & .015 & .102 & .067 & -.089 \\
\hline & .180 & .740 & .144 & .608 & .162 \\
\hline How many Vitamin A doses to be given to child? & .156 & .041 & -.006 & .353 & -.215 \\
\hline & .064 & .676 & .966 & .208 & .111 \\
\hline From when should Vitamin A dose be started? & -.027 & -.049 & .050 & -.033 & .076 \\
\hline & .653 & .484 & .639 & .868 & .432 \\
\hline What happens when diarrhea occurs? & .009 & .026 & -.047 & -.073 & .073 \\
\hline & .685 & .288 & .212 & .306 & .032 \\
\hline What should be given to a baby below 6 months with & -.038 & -.040 & .045 & .138 & -.331 \\
\hline diarrhea? & .624 & .665 & .744 & .507 & .009 \\
\hline How to prepare ORS? & -.119 & .271 & -.195 & .277 & .136 \\
\hline & .058 & .000 & .080 & .187 & .179 \\
\hline What is the RDK kit for? & -.047 & .077 & .119 & -.061 & .036 \\
\hline & .188 & .064 & .057 & .606 & .528 \\
\hline What to do for prevention of malaria? & .159 & .148 & -.322 & .804 & .118 \\
\hline & .179 & .282 & .123 & .042 & .533 \\
\hline Where to spread DDT? & .014 & .216 & .140 & .165 & -.131 \\
\hline & .862 & .024 & .332 & .554 & .317 \\
\hline How to detect malaria? & -.037 & -.074 & .083 & -.036 & -.031 \\
\hline & .202 & .028 & .104 & .712 & .502 \\
\hline Whom to discuss with on VHND? & .093 & .069 & -.313 & .315 & -.280 \\
\hline & .250 & .461 & .028 & .241 & .031 \\
\hline
\end{tabular}

South East Asia Journal of Public Health 2015;5(2):57-64 
Colostrum feeding to newborn, importance of birth certificate and how to get it done, ORS preparation, RDK kit, how to detect malaria, importance of DDT spray and where to spray. Therefore, education level is very much important to know and practice the key activities related to maternal and child health along common illnesses. As per the guidelines the minimum qualification of ASHAs should be minimum class VIII. It has been seen that most of the ASHAs are above class $\mathrm{X}$ standard. But at the same time around $8 \%$ ASHAs have been found below class VIII standard. As educational level has significant positive effect on KAP status, minimum qualification of ASHAs should be at least class $\mathrm{X}$ standard. There may be some relaxation where it is difficult to get such educated women.

Communication media like TV, radio and mobile have also been found to have significant effect on certain KAP by ASHAs. For, example, TV shows significant effect on early and exclusive breastfeeding. Similarly, Radio shows impact on TT vaccines during pregnancy, danger signs of pregnancy, early initiation of breastfeeding, complementary feeding, newborn care (specially restricting bath to newborn), birth certificate, malaria prevention, etc. National Rural Health Mission (NRHM), Assam launched an ASHA radio programme which is based on infotainment i.e. information through entertainment. ${ }^{11}$ The programme has a drama with characters like ASHA, an older lady, teacher, etc. The theme song is also very encouraging and all ASHAs could even remember each and every line of the song. In this context, ASHAs were provided a radio to listen to the ASHA radio programme. It has been found that communication media like TV, Radio, Mobile etc has significant positive effect on KAP status of ASHAs in various components. Similar studies support this statement. ${ }^{12,13}$ So, this kind of innovative message dissemination techniques might be helpful for increasing the knowledge and practice level of frontline workers like ASHAs.

Gathering information from other frontline workers, ANMs, doctors through mobile phone also showed significant effect on the KAP status on certain points like, danger signs of pregnancy, importance of institutional delivery, Infant and Young Child Feeding (IYCF) practices, newborn care, importance of birth certificate and how to acquire it, management of diarrhoea and quality Village Health and Nutrition Day (VHND) organization. Getting information through mobile is very easy and prompt. So, frontline workers like ASHAs usually build network through mobiles. Information may also be shared through mobile phones in local languages for increasing the knowledge level of frontline workers. Frontline workers or community health workers like ASHAs can play an important role in reducing infant mortality rate (IMR) and Under Five mortality rate including Maternal Mortality Ratio (MMR). ${ }^{1}$ Various trials have shown substantial reductions in child mortality, particularly with case management of ill children by community health workers, like a meta-analysis of community-based trials of the effect of case management of pneumonia on mortality in neonates, infants, and preschool children suggested an overall reduction of $24 \%$ in mortality. ${ }^{14}$ Again, a trial conducted in Ethiopia on training of local coordinators to teach mothers to give anti-malarial promptly to their sick children in the home showed $40 \%$ reduction in under-5 mortality. ${ }^{15}$ Another systematic review of the management of pneumonia and malaria by community health workers identified seven intervention models according to their role in assessment of children, system of referral to health facility (verbal or written), and location of the drug stock. ${ }^{16} \mathrm{~A}$ controlled trial in rural India showed that home-based neonatal care and management of sepsis can more than halve neonatal mortality in a high-mortality setting. ${ }^{17}$ All the findings suggest that the proper training, re-orientation and proper dissemination of messages through different suitable medium would be required to reach our goals in proper sense.

The health systems in many countries are too weak and uneven to enable the scaling-up of essential interventions for maternal, newborn, and child health ${ }^{18}$ and one key challenge is the need to develop and strengthen human resources to deliver essential interventions. $^{19,20}$ The density of health workers (doctors, nurses, midwives) is inversely associated with maternal, infant, and under-5 mortality, ${ }^{21}$ Policymakers generally refer to Community Health Workers as "volunteers" or "activists"; which means self-motivated persons working willingly for the development of their own community and without any anticipation in monetary terms. This concept has got significant support that culminate in the nationwide programme of ASHA under NRHM. ${ }^{2}$ The Ministry of Health \& Family Welfare (MoHFW) developed a 23-day training schedule in four phases to provide the necessary knowledge \& skills to ASHAs. However, data shows that the quantity and quality of the training in practice must be improved in order to improve the performance of ASHAs. ${ }^{9}$ As ASHAs are the first contact point at grass root level, they play a key role in disseminating any health messages to the rural mass and help the population to avail the health care services on time. This has a positive impact on overall improvement in the health status of the community and also helps in reducing maternal, infant and child mortality and morbidity. Therefore, re-orientation on the subjects and programmes through quality training and making them aware with the help of communication media will facilitate to achieve the broad objective of the health mission in developing countries like India. However, in the present study, responses of the ASHAs could have been classified more accurately if more categories were considered, namely, very poor, poor, average, good and very good.

\section{Conclusion}

From the present study, it has been observed that most of the ASHAs were competent in some aspects of health and nutrition issues which have direct impact on child survival and maternal health. But at the same time average to poor knowledge level have been observed in 
some other important indicators. For improving the KAP of ASHAs, they should be properly trained on the various aspects of KAP. Moreover timely re-orientation and message and information sharing through different suitable medium like TV, Radio, mobile etc. would be helpful. Though there are provisions for training of ASHAs on different modules containing important aspects of health and nutrition, still there is lacunae left in their knowledge regarding the various aspects of morbidity and mortality. Training of ASHAs seems to be neither as per norms and not conducted at a regular basis. Training is the backbone of capacity building and functioning of ASHAs. So it must be done timely, properly and effectively. Frequency and quality of training for ASHA workers must be strengthened. Providing incentives timely might be another motivating factor for them to work hard and work sincerely. Most of the ASHAs expect a monthly salary instead of incentives. All these aspects should be kept in the agenda of discussion of policymakers when there is a question of achieving Millennium Development Goals (MDGs) or in broader aspects achieving Sustainable Development Goals within the timeframe.

\section{References}

1. frontlinehealthworkers.org. No Health Workers, No Health. Frontline Health Workers Coalition 2012:Jan:2-3

2. Sujay RJ, George M. Healthcare through Community Participation-Role of ASHAs. Econ Polit Weekly 2010:47(10):70-6

3. Government of India. NRHM-ASHA Module Guidelines, Ministry of Health and Family Welfare, New Delhi. 2005.

4. Shrivastava SR, Shrivastava PS. Evaluation of trained Accredited Social Health Activist (ASHA ) workers regarding their knowledge, attitude and practices about child health. Rural Remote Health 2012. 12(4): 1-7.

5. Mahyavanshi DK, Patel MG, Kartha G, Purani SK, Nagar SS. A cross sectional study of the knowledge, attitude and practice of ASHA workers regarding child health (under five years of age) in Surendranagar district. Healthline 2011;2 (2): 50-3.

6. Mohapatra B, Datta U, Gupta S, Tiwari VK, Nair $\mathrm{KS}$, Adhish $\mathrm{V}$ et al. An assessment of the functioning and impact of Janani Suraksha Yojana in Orissa. Health Popul Perspect Issues 2008;31(2):120-5.

7. Swain S, Swain P, Nair KS, Dhar N, Gupta S, Nandan D. A rapid appraisal of functioning of ASHA under NRHM in Orissa. Health Popul Perspect Issues 2008;31(2):73-9.

8. Berman PA, Gwatkin DR, Burger SE. Community-based health workers: head start or false start towards health for all? Soc Sci Med 1987; 25:443-59.
9. Bajpai N, Dholakia RH. Improving the performance of accredited social health activists in India. Working paper no. 1, New York, NY: Columbia University; 2011.

10. Ministry of Health \& Family Welfare, Govt. of India. National Rural Health Mission. http:// nrhm.gov.in/communitisation/asha/asha-supportmechanism/supporting-mechanism.html (accessed 15 March 2016)

11. National Rural Health Mission (NRHM). ASHA Radio Programme under NRHM, Assam. http:// www.nrhmassam.in/asharadio.php (accessed 16 March 2016)

12. Pallikadavath S, Sreedharan C, Stones RW. Sources of AIDS awareness among women in India. AIDS care 2006;18(1): 44-8.

13. Garai A, Ganesan R. Role of Information and Communication Technologies in Accelerating the Adoption of Healthy Behaviors. $J$ Fam Welfare 2010; 56:109-18.

14. Sazawal S, Black RE. Effect of pneumonia case management on mortality in neonates, infants, and preschool children: a meta-analysis of community-based trials. Lancet Infect Dis 2003;3:547-56.

15. Kidane G, Morrow RH. Teaching mothers to provide home treatment of malaria in Tigray, Ethiopia: a randomised trial. Lancet 2000; 356: 550-5.

16. Winch PJ, Gilroy KE, Wolfheim C, Starbuck ES, Young MW, Walker LD, Black RE. Intervention models for the management of children with signs of pneumonia or malaria by community health workers. Health Policy Plan 2005;20:199-212.

17. Bang AT, Bang RA, Baitule SB, Reddy $\mathrm{MH}$, Deshmukh MD. Effect of home-based neonatal care and management of sepsis on neonatal mortality: field trial in rural India. Lancet 1999;354:1955-61.

18. Travis $\mathrm{P}$, Bennett $\mathrm{S}$, Haines A, et al. Overcoming health-systems constraints to achieve the Millennium Development Goals. Lancet 2004; 364: 900-6.

19. World Health Organization. Working together for Health: World Health Report. Geneva; WHO, 2006.

20. Chen L, Evans T, Anand S, et al. Human resources for health: overcoming the crisis. Lancet 2004;364:1984-90.

21. Anand S, Barnighausen T. Human resources and health outcomes. Lancet. 2004; 364: 1603-9.Use of the short-form-36 health survey to assess quality of life among Australian elderly. Aus $J$ Ageing 1996;15(3):132-5. 